\title{
ABUNDANCE OF REAL LINES ON REAL PROJECTIVE HYPERSURFACES
}

\author{
S. Finashin, V. Kharlamov
}

There is geometry in the humming of the strings. There is music in the spacing of the spheres.

PYTHAGORAS (6th century BC)

\section{Revelations}

Our aim is to show that in the case of a generic real hypersurface $X$ of degree $2 n-1$ in a projective space of dimension $n+1$ the number $\mathscr{N}_{\mathbb{R}}$ of real lines on $X$ is not less than approximately the square root of the number $\mathscr{N}_{\mathbb{C}}$ of complex lines. More precisely, $\mathscr{N}_{\mathbb{R}} \geq(2 n-1)$ !!, while due to Don Zagier $[\mathrm{GM}] \mathscr{N}_{\mathbb{C}} \sim \sqrt{\frac{27}{\pi}}(2 n-1)^{2 n-\frac{3}{2}}$, so that

$$
\log \mathscr{N}_{\mathbb{R}} \geq n \log 2 n+O(n)=\frac{1}{2} \log \mathscr{N}_{\mathbb{C}}
$$

Note that $\mathscr{N}_{\mathbb{R}}$, unlike $\mathscr{N}_{\mathbb{C}}$, depends not only on $n$ but also on the choice of $X$. The key point of our estimate is an appropriate signed count of the real lines that makes the sum invariant. This sum, which we denote by $\mathscr{N}_{\mathbb{R}}^{e}$, is nothing but the Euler number of a suitable vector bundle (see 3.1). Its evaluation gives finally the conclusion:

$$
\mathscr{N}_{\mathbb{R}} \geq \mathscr{N}_{\mathbb{R}}^{e}=(2 n-1) ! !
$$

We came to it in 2010-2011, when we started working on a survey on real cubic hypersurfaces. Realizing that Segre's division of real lines on cubic surfaces in two species, elliptic and hyperbolic, leads to a remarkable relation $h-e=3$ between the number of hyperbolic lines, $h$, and number $e$ of elliptic, we looked for conceptual explanations and generalizations.

Later, we recollected that the non-vanishing of the Stiefel-Whitney number of the same bundle (which implies existence of real lines) was proved much earlier by O. Debarre and L. Manivel in [DM, and that the Euler number was used by J. Solomon in his thesis [5] to perform a signed count of real lines on quintic 3folds. In September 2011 we were informed by C. Okonek and A. Teleman that they obtained the bound (1.0.1) and that they followed a similar approach. It is when an account of their proof was posted on arXiv that we decided to compose this note and to present our proof (in our opinion, a bit simpler and more geometric) and a few related observations, like disclosing precise relation to Welschinger's indices, see 5.2, and revealing the "secret" of the "magic formula" $h-e=3$, see 4.1.1.

2000 Mathematics Subject Classification. 14N15, 14P25, 14J80.

The second author was partially funded by the ANR-09-BLAN-0039-01 grant of Agence Nationale de la Recherche, and is a member of FRG Collaborative Research "Mirror Symmetry \& Tropical Geometry" (Award No. 0854989). 


\section{Origin of Symmetry}

2.1. The polar correspondence. The Grassmannian $\widetilde{G}_{2}\left(\mathbb{R}^{n+2}\right)$ of oriented 2planes can be canonically identified with the purely imaginary quadric $Q^{n}(\mathbb{C})$ defined in $P^{n+1}(\mathbb{C})$ by equation $x_{0}^{2}+x_{1}^{2}+\cdots+x_{n+1}^{2}=0$. Namely, a real oriented plane $p \subset \mathbb{R}^{n+2}$ defines a real oriented line $\ell_{\mathbb{R}} \subset P^{n+1}(\mathbb{R})$, which decomposes its complexification $\ell_{\mathbb{C}} \subset P^{n+1}(\mathbb{C})$ into a pair of halves permuted by the complex conjugation. Each half intersects $Q^{n}(\mathbb{C})$ at a single point. We give privilege to that half, $h_{l}$, whose complex orientation bounds the given orientation of $\ell_{\mathbb{R}}$. The map $p \mapsto q=Q^{n}(\mathbb{C}) \cap h_{l}$ yields a diffeomorphism $\varkappa: \widetilde{G}_{2}\left(\mathbb{R}^{n+2}\right) \rightarrow Q^{n}(\mathbb{C})$, which we call the polar correspondence.

In the reverse direction, the polar correspondence can be described as follows. A point $q \in Q^{n}(\mathbb{C}) \subset P^{n+1}(\mathbb{C})$ is represented by a complex line $L_{q} \subset \mathbb{C}^{n+2}$, the complex line $L_{q}$ projects to $\mathbb{R}^{n+2}$ into a real plane $p$ by averaging $v \mapsto \frac{1}{2}(v+\bar{v})$, and the complex orientation of $L_{q}$ is pushed forward to orient the plane $p$.

This yields the following interpretation for the tautological bundles of the oriented Grassmannian, $\widetilde{G}_{2}\left(\mathbb{R}^{n+2}\right)$, and of the non-orientable one, $G_{2}\left(\mathbb{R}^{n+2}\right)$.

2.1.1. Proposition. The polar correspondence $\widetilde{G}_{2}\left(\mathbb{R}^{n+2}\right) \rightarrow Q^{n}(\mathbb{C})$ identifies the tautological (oriented 2-plane) bundle $\widetilde{\tau}_{2, n+2}: \widetilde{E}_{2}\left(\mathbb{R}^{n+2}\right) \rightarrow \widetilde{G}_{2}\left(\mathbb{R}^{n+2}\right)$ with the restriction, $\tau_{n}: E^{n}(\mathbb{C}) \rightarrow Q^{n}(\mathbb{C})$, to $Q^{n}(\mathbb{C})$ of the tautological (complex line) bundle on $P^{n+1}(\mathbb{C})$. This yields identification of the tautological bundle $\tau_{2, n+2}$ over $G_{2}\left(\mathbb{R}^{n+2}\right)$ with the quotient of $\tau_{n}$ by the complex conjugation.

2.2. Characteristic classes. The polar correspondence leads to a simple explicit formula for the Euler class, $e\left(S \mathrm{~m}^{2 m-1}(F)\right)$, of symmetric powers of the dual tautological bundles, $F=\widetilde{\tau}_{2, n+2}^{*}$. Namely, the polar correspondence identifies $F$ with the real 2-bundle underlying complex line bundle $L=\varkappa^{*}\left(\tau_{n}^{*}\right)$, so, $F \otimes \mathbb{C}=L \oplus \bar{L}$, and $\operatorname{Sym}^{k}(F) \otimes \mathbb{C}=\operatorname{Sym}^{k}(F \otimes \mathbb{C})=L^{k} \oplus L^{k-1} \bar{L} \cdots \oplus \bar{L}^{k}$, for any $k \geq 1$. The complex conjugation interchanges $L^{a} \bar{L}^{b}$ with $L^{b} \bar{L}^{a}$, thus, for any odd $k=2 m-1$, the real vector bundle $\operatorname{Sym}^{2 m-1}(F)$ is the real part of $L^{2 m-1} \oplus L^{2 m-2} \bar{L} \oplus \cdots \oplus$ $\bar{L}^{2 m-1}$. We define an isomorphism between $L^{2 m-1} \oplus L^{2 m-2} \bar{L} \oplus \cdots \oplus L^{m} \bar{L}^{m-1}$ and $\operatorname{Sym}^{2 m-1}(F)$ by projecting $v \mapsto \frac{1}{2}(v+\bar{v})$. It transports the complex orientation of $L^{2 m-1} \oplus L^{2 m-2} \bar{L} \oplus \cdots \oplus L^{m} \bar{L}^{m-1}$ to an orientation of $\operatorname{Sym}^{2 m-1}(F)$, so that the Euler class of the latter becomes well defined and equal to the Chern class of the former one.

2.2.1. Proposition. Under the above orientation convention,

$e\left(\operatorname{Sym}^{2 m-1}(F)\right)=c_{m}\left(L^{2 m-1} \oplus L^{2 m-2} \bar{L} \oplus \cdots \oplus L^{m} \bar{L}^{m-1}\right)=(2 m-1) ! c_{1}(L)^{m}$.

\section{Absolution}

3.1. Euler's numbers. By Proposition 2.1.1, the polar correspondence identifies the total space $E$ of the bundle $\operatorname{Sym}^{2 n-1}\left(\tau_{2, n+2}^{*}\right)$ with the quotient by complex conjugation of the total space of a complex $n$-bundle $\left(\tau_{n}^{*}\right)^{2 n-1} \oplus\left(\tau_{n}^{*}\right)^{2 n-2}\left(\bar{\tau}_{n}^{*}\right) \oplus \cdots \oplus$ $\left(\tau_{n}^{*}\right)^{n}\left(\bar{\tau}_{n}^{*}\right)^{n-1}$ over a complex n-manifold, $Q^{n}(\mathbb{C})$. Since any anti-holomorphic involution on a complex manifold of even complex dimension preserves the orientation, the manifold $E$ inherits an orientation.

Let $\mathscr{N}_{\mathbb{R}}^{e}$ denote the Euler number of $\operatorname{Sym}^{2 n-1}\left(\tau_{2, n+2}^{*}\right)$ with respect to this orientation. Given a section $s: G_{2}\left(\mathbb{R}^{n+2}\right) \rightarrow E$ and an isolated zero $x$ of $s$, let $I_{x}^{e}(s)$ 
denote its index, that is the local Euler number of $s$ at $x$. If $s$ has only isolated zeros, then $\mathscr{N}_{\mathbb{R}}^{e}=\sum_{x} I_{x}^{e}(s)$, and we let $\mathscr{N}_{\mathbb{R}}(s)=\sum_{x}\left|I_{x}^{e}(s)\right|$.

\subsubsection{Theorem.}

(1) $\mathscr{N}_{\mathbb{R}}^{e}=(2 n-1) !$ !

(2) For any section $s$ having only isolated zeros, $\mathscr{N}_{\mathbb{R}}(s) \geq(2 n-1)$ !!.

Proof. By definition, twice $\mathscr{N}_{\mathbb{R}}^{e}$ equals the Euler number of $S y m^{2 n-1}\left(\widetilde{\tau}_{2, n+2}^{*}\right)$, which in accordance with Proposition 2.2 .1 is equal to $(2 n-1) ! ! c_{1}\left(\tau_{n}^{*}\right)^{n}\left[Q^{n}(\mathbb{C})\right]$, while $c_{1}\left(\tau_{n}^{*}\right)^{n}\left[Q^{n}(\mathbb{C})\right]=\operatorname{deg} Q^{n}=2$. This gives (1), wherefrom (2) is straightforward.

Consider a real homogeneous polynomial $f \in H^{0}\left(P^{n+1}, \mathcal{O}(d)\right)$ defining a real hypersurface $X$ of degree $d=2 n-1$ in $P^{n+1}$. Restricting $f$ to planes $p \subset \mathbb{R}^{n+2}$, we obtain a section $s_{f}$ of $S y m^{2 n-1}\left(\tau_{2, n+2}^{*}\right)$. Its zeros represent real lines lying on $X$. We call a real line $\ell \subset X$ isolated if it represents an isolated zero of $s_{f}$, and attribute to it the real multiplicity equal, by definition, to $\left|I_{\ell}^{e}\left(s_{f}\right)\right|$. Thus, Theorem 3.1.1 implies the following result.

3.1.2. Corollary. Assume that all the real lines $\ell \subset X$ of a real hypersurface $X \subset P^{n+1}$ of degree $2 n-1$ are isolated. Then $\sum_{\ell \subset X}\left|I_{\ell}^{e}\left(s_{f}\right)\right| \geq(2 n-1) ! !$.

As is known (see, for example, BVV), for a generic $f$ (that is for a generic $X$ ) the section $s_{f}$ is transverse to the zero section, and so, all the lines $\ell \subset X$ are isolated and all have indices $I_{\ell}^{e}\left(s_{f}\right)= \pm 1$. Let $\mathscr{N}_{\mathbb{R}}^{+}$and $\mathscr{N}_{\mathbb{R}}^{-}$denote the number of real lines of indices 1 and -1 , respectively. Thus, for a generic $f$, the number of real lines is

$$
\mathscr{N}_{\mathbb{R}}^{+}+\mathscr{N}_{\mathbb{R}}^{-} \geq \mathscr{N}_{\mathbb{R}}^{+}-\mathscr{N}_{\mathbb{R}}^{-}=\mathscr{N}_{\mathbb{R}}^{e}=(2 n-1) ! !
$$

3.1.3. Corollary. For a generic hypersurface $X \subset P^{n+1}$ of degree $(2 n-1)$, the number of real lines $\ell \subset X$ is finite and bounded from below by $(2 n-1) ! !$.

Remark. Our choice of an orientation of $E$ and of the multiplicities of $\ell \subset X$ is a bit different from that in OT. The orientation is not essential for us, since $\left|\mathscr{N}_{\mathbb{R}}{ }^{e}\right|$ and $\left|I_{x}^{e}(s)\right|$ remain invariant, while the multiplicities make a difference. In fact, the complex multiplicities used in OT] are bounded from below by our indices $\left|I_{x}^{e}(s)\right|$.

\section{SHOwBIZ}

4.1. Cubic surfaces: 3 versus 27. Recall, that any nonsingular cubic surface contains exactly $27=c_{4}\left(\operatorname{Sym}^{3}\left(\tau_{2,4}^{*}(\mathbb{C})\right)\right)\left[G_{2}\left(\mathbb{C}^{4}\right)\right]$ complex lines $\left(\right.$ here $\tau_{2,4}(\mathbb{C})$ denotes the tautological bundle over the complex Grassmannian $G_{2}\left(\mathbb{C}^{4}\right)$ ), and it implies, in particular, that for a nonsingular cubic surface, a section $s_{f}$, which can be defined for $\operatorname{Sym}^{3}\left(\tau_{2,4}^{*}(\mathbb{C})\right)$ as well as for $\operatorname{Sym}^{3}\left(\tau_{2,4}^{*}\right)$, is in the both cases transversal to the zero section. As a result, Theorem 3.1.1 reads as identity $\mathscr{N}_{\mathbb{R}}^{e}=\mathscr{N}_{\mathbb{R}}^{+}-\mathscr{N}_{\mathbb{R}}^{-}=3$ and estimate $\mathscr{N}_{\mathbb{R}} \geq 3$.

The estimate $\mathscr{N}_{\mathbb{R}} \geq 3$ was known already to Schläfli $[\mathrm{Sc}]$, who proved that the number of real lines on a non-singular real cubic surface takes only the values $27,15,7$, and 3 . Segre $[\mathrm{Se}]$ was probably the first to observe that the real lines on a nonsingular real cubic surface can be divided in two species, named by him hyperbolic and elliptic, and that numerically the distribution between the species, $\mathscr{N}_{\mathbb{R}}=h+e$, is as follows: $27=15+12,15=9+6,7=5+2$, and $3=3+0$. It implies the following numerical coincidence. 
4.1.1. Proposition. For any non-singular real cubic surface,

$$
h=\mathscr{N}_{\mathbb{R}}^{+}, \quad e=\mathscr{N}_{\mathbb{R}}^{-} .
$$

4.2. A direct proof of Proposition 4.1.1. Consider a real line $\ell$ on a real nonsingular projective cubic surface $X$. Choose projective coordinates $x, y, u, v$ in $P^{3}$ so that the line $\ell$ is given by equations $x=y=0$. Then the defining polynomial of $X$ has the form $f=u^{2} L_{11}+2 u v L_{12}+v^{2} L_{22}+u Q_{1}+v Q_{2}+C$, where $L_{i j}$, $Q_{i}$, and $C$ are homogeneous polynomials in $x$ and $y$ of degree, respectively, one, two, and three. According to Segre's definitions, the line $\ell$ is elliptic (respectively, hyperbolic) if the quadratic form $L_{12}^{2}-L_{11} L_{22}$ is definite (respectively, indefinite).

4.2.1. Lemma. A real line contributes 1 into $\mathscr{N}_{\mathbb{R}}^{+}$if the line is hyperbolic, and to $\mathscr{N}_{\mathbb{R}}^{-}$if elliptic.

Proof. A line $\ell^{\prime}$ in a neighborhood of $\ell \in G_{2}\left(\mathbb{R}^{4}\right)$ hits the coordinate projective planes $v=0$ and $u=0$ at some points $\left[x_{1}: y_{1}: 1: 0\right]$ and $\left[x_{2}: y_{2}: 0: 1\right]$, and thus, $\ell^{\prime}$ can be given in a parametric form as $(u, v) \mapsto u\left(x_{1}, y_{1}, 1,0\right)+v\left(x_{2}, y_{2}, 0,1\right)$. The value of section $s_{f}\left(\ell^{\prime}\right)$ is defined by the restriction of $f$ to $\ell^{\prime}$, i.e, by substitution $x=u x_{1}+v x_{2}$ and $y=u y_{1}+v y_{2}$ in the polynomial $f$. Letting $L_{i j}=l_{i j} x+m_{i j} y$ for all $i, j$, we obtain $s_{f}\left(\ell^{\prime}\right)=u^{2}\left[l_{11}\left(u x_{1}+v x_{2}\right)+m_{11}\left(u y_{1}+v y_{2}\right)\right]+\ldots$ It is straightforward to check, following the polar correspondence, that our orientation of the total space of $\operatorname{Sym}^{3}\left(\tau_{2,4}^{*}\right)$ agrees with the order $x_{1}, x_{2}, y_{1}, y_{2}$ of the local coordinates in $G_{2}\left(\mathbb{R}^{4}\right)$ and the frame $u^{3}, u^{2} v, u v^{2}, v^{3}$ that gives a local trivialization of $\operatorname{Sym}^{3}\left(\tau_{2,4}^{*}\right)$. The Jacobi matrix of $s_{f}$ in these coordinates is as follows

$$
\left[\begin{array}{cccc}
l_{11} & 0 & m_{11} & 0 \\
2 l_{12} & l_{11} & 2 m_{12} & m_{11} \\
l_{22} & 2 l_{12} & m_{22} & 2 m_{12} \\
0 & l_{22} & 0 & m_{22}
\end{array}\right]
$$

Now, it remains to notice that the determinant of this matrix, whose sign is the index $I_{\ell^{\prime}}^{e}\left(s_{f}\right)$, is opposite to the discriminant of the binary quadratic form $L_{12}^{2}-$ $L_{11} L_{22}$.

\section{Black Holes}

5.1. Congruences. As was disclosed by Grünberg and Moree [GM], the residues of $\mathscr{N}_{\mathbb{C}}$ modulo $2^{q}$ form a $2^{q}$-periodic sequence for all $q \geq 1$. For the modulo $2^{q}$ residues of $\mathscr{N}_{\mathbb{R}}^{e}=(2 n-1)$ !! the $2^{q}$-periodicity is evident. Up to $q=2$ the two sequences of residues coincide, $\mathscr{N}_{\mathbb{C}}=\mathscr{N}_{\mathbb{R}}^{e} \bmod 4$. The relation between 8-residues is less trivial; the period is $1,1,3,3,5,5,7,7$ for $\mathscr{N}_{\mathbb{C}}$ and $1,1,3,7,1,1,3,7$ for $\mathscr{N}_{\mathbb{R}}^{e}$. What can be general rules and conceptual explanations?

In the case of cubic surfaces another congruence holds, $\mathscr{N}_{\mathbb{R}}=\mathscr{N}_{\mathbb{C}} \bmod 4$. However, for any fixed dimension $n \geq 3$, the range of $\mathscr{N}_{\mathbb{R}}$ includes all the odd integers in a certain interval from some $\mathscr{N}_{\mathbb{R}}^{\text {min }} \geq \mathscr{N}_{\mathbb{R}}^{e}$ to some $\mathscr{N}_{\mathbb{R}}^{\text {max }} \leq \mathscr{N}_{\mathbb{C}}$. In a sense, this phenomenon reflects the difference in the Galois groups of the corresponding enumerative problems (starting from 3-folds the Galois group is the full symmetric group, see $[\mathbb{H}$ ). 
5.2. Welschinger invariants. There is a method, introduced by J.Y. Welschinger $\mathrm{We}$, of counting real rational curves in a given homology class on arbitrary real rational surfaces and certain real 3-folds. As was observed by J.Solomon [S0], in the case of quintic 3 -folds the number $\mathscr{N}_{\mathbb{R}}^{e}=15$ can be seen as the Welschinger invariant of the generator in the second homology group of the complexification of the quintic. To get $\mathscr{N}_{\mathbb{R}}^{e}=3$ in the case of cubic surfaces, one should take the sum of the Welschinger invariants over all homology classes of projective degree 1 . Though, the invariants should be taken not with the signs traditionally applied in the case of rational surfaces, but with signs copied from that used in the case of 3 -folds (just consider the Pin $^{-}$-structure $q: H_{1}\left(X_{\mathbb{R}} ; \mathbb{Z} / 2\right) \rightarrow \mathbb{Z} / 4$ inherited by the real locus $X_{\mathbb{R}}$ of a non-singular cubic surface from the ambient real projective space and define the sign of a line $l$ to be equal to $\left.i^{q(l)-1}\right)$. As an additional similarity, let us point that Welschinger invariants tend to have asymptotic and arithmetic properties analogous to that of the Euler numbers of real lines treated in this note (see, for example, [IKS]). Is there a general theory of real enumerative invariants that bring all these results together?

5.3. Hypersurfaces of other degrees. If $X$ is a real hypersurface of odd degree $d<2 n-1$ in $P^{n+1}(\mathbb{R})$, the dimension of the variety $F_{\mathbb{R}}(X)$ of real lines contained in $X$ is $\geq 2 n-1-d$, and is equal to $2 n-1-d$ for generic $X$; in particular, it is always nonempty (see DM]). What is about abundance? Is there a lower bound for the total Betti number, $b_{*}\left(F_{\mathbb{R}}(X)\right)$ comparable in logarithmic scale with $b_{*}\left(F_{\mathbb{C}}(X)\right)$ ?

\section{REFERENCES}

[BVV] W. Barth, A. Van de Ven, Fano varieties of lines on hypersurfaces, Arch. Math. (Basel) 31 (1978/79), 96-104.

[DM] O. Debarre, L. Manivel, Sur les intersections complètes réelles, CRAS 331 (Série I) (2000), 887-992.

[GM] D.B. Grünberg, P. Moree, Sequences of enumerative geometry: congruences and asymptotics (with Appendix by Don Zagier), Experiment. Math. 17 (2008), 409-426.

[H] J. Harris, Galois groups of enumerative problems, Duke Math. J. 46 (1979), 685-724.

[IKS] I. Itenberg, V. Kharlamov, E. Shustin, Welschinger invariants of real Del Pezzo surfaces of degree $\geq 3$, arXiv:1108.3369, 32 pages.

[OT] C. Okonek, A. Teleman, Intrinsic signs and lower bounds in real algebraic geometry, arXiv:1112.3851, 17 pages.

[Sc] L.Schläfli, An attempt to determine the twenty-seven lines upon a surface of the third order, and to divide such surfaces into species in reference to the reality of the lines upon the surface, Quart. J. Pure Appl. Math. 2 (1858), 110-120.

[Se] B. Segre, The Non-Singular Cubic Surfaces. A new method of Investigation with Special Reference to Questions of Reality, Oxford Univ. Press, London, 1942.

[So] J.P. Solomon, Intersection theory on the moduli space of holomorphic curves with Lagrangian boundary conditions, arXiv:math/0606429, 79 pages.

[We] J.-Y. Welschinger, Invariants of real rational symplectic 4-manifolds and lower bounds in real enumerative geometry, C. R. Acad. Sci. Paris, Sér. I 336 (2003), 341-344.

Middle East Technical University, Department of Mathematics

ANKARA 06531 TuRKEy

Université de Strasbourg Et IRMA (CNRS)

7 rue René Descartes 67084 Strasbourg Cedex, France 